\title{
Effects of acculturation, coping strategies, locus of control, and self-efficacy on chronic pain: study of Chinese immigrant women in Italy - insights from a thematic field analysis
}

\author{
This article was published in the following Dove Press journal: \\ Journal of Pain Research \\ 6 June 2017 \\ Number of times this article has been viewed
}

\section{Tania Simona Re' \\ Nicola Luigi Bragazzi ${ }^{1,2}$ \\ Anna Siri' \\ César Cisneros Puebla ${ }^{3}$ \\ Susanne Friese ${ }^{4}$ \\ Mário Simões ${ }^{5}$ \\ Joël Candau 6 \\ Hicham Khabbache ${ }^{7}$ \\ 'UNESCO Chair "Health Anthropology, Biosphere and Healing Systems", Genoa, Italy; ${ }^{2}$ Department of Health Sciences (DISSAL), School of Public Health, University of Genoa, Genoa, Italy; ${ }^{3}$ Department of Sociology, Metropolitan Autonomous University-Iztapalapa, San Rafael Atlixco, Mexico; ${ }^{4}$ Max Planck Institute for the Study of Religious and Ethnic Diversity, Göttingen, Germany; ${ }^{5}$ Medical Faculty, University of Lisboa, Lisboa, Portugal; ${ }^{6}$ Laboratory of Anthropology and Cognitive and Social Psychology (LAPCOS), University of Nice-Sophia Antipolis, Nice, France; ${ }^{7}$ Faculty of Literature and Humanistic Studies, Sidi Mohamed Ben Abdellah University, Fez, Morocco}

Correspondence: Tania Simona Re UNESCO Chair "Health Anthropology, Biosphere and Healing Systems", Via Balbi,

5, 16126, Genoa, Italy

Tel +3910 $2099745 \times 5987$

Email tania.re77@gmail.com

Nicola Luigi Bragazzi

Department of Health Sciences (DISSAL),

School of Public Health, University of

Genoa, Via Antonio Pastore I, Genoa

16132, Italy

Tel +390l 03537664

Fax +390103537669

Email robertobragazzi@gmail.com
Abstract: Chronic pain represents a common public health concern worldwide. It is a complex phenomenon, owing to the interaction of different factors, including biological, physiological, psychological, environmental, and social variables. Some groups, such as women and immigrants, are particularly vulnerable. However, little is known about how Chinese women in Italy live with and face chronic pain. The present study aimed at filling this knowledge gap by examining the burden of chronic pain in Chinese immigrants in Italy in terms of acculturation processes, perceived control over disease, social networks, and coping strategies. A qualitative approach was used, performing a thematic field analysis. We interviewed 82 Chinese women from different Italian towns (Genoa, Milan, Turin, Bologna, Florence, and Prato) in depth. The sense of belonging to the host culture was strong in our sample. However, this did not simply reflect or translate into a linear engagement with medical systems, as health care pathways were more complex and dual (both Chinese and Western). Chinese women who felt deeply rooted in the Italian environment did not discontinue the use of traditional Chinese medicine. Chronic pain extensively and adversely affected daily life, particularly interfering with work. Coping strategies were mainly adaptive behaviors, being problem focused or maladaptive, relying upon "cope and avoid" mechanisms. Chinese women preferred to use traditional Chinese remedies rather than conventional medicine, while using the Italian system in emergencies. Perceived control over chronic pain was usually external. Finally, Chinese women with chronic pain benefit from social networks and support, which were mainly composed of Chinese peers. In conclusion, our findings underline the tremendous burden of chronic pain affecting all aspects of Chinese women's lives. Health care workers and providers should be aware of the complexity of chronic pain Therefore, a holistic approach, involving different stakeholders, should be adopted when managing chronic pain and approaching immigrant patients.

Keywords: chronic pain, Chinese immigrants, qualitative research, thematic field analysis

\section{Introduction}

According to the International Association for the Study of Pain (IASP), pain is defined as "an unpleasant sensory and emotional experience associated with actual or potential tissue damage, or described in terms of such damage". ${ }^{1}$ This definition summarizes both the mental and physical components of the pain, emphasizing that pain is a multidimensional experience and is not limited to a feeling or sensation in a single or multiple parts of the body. There is currently no universal consensus about the definition of chronic 
pain: some authors define it as a form of pain lasting longer than 3 months, whereas others adopt other duration criteria ( 6 or even 12 months). ${ }^{2}$ Chronic pain undoubtedly represents a common public health concern worldwide, constituting "a burden to individuals and a challenge to society". ${ }^{3}$ It is a complex phenomenon, owing to the interaction of different factors, including biological, physiological, psychological, environmental, and social variables, and thus, it requires a multidisciplinary approach. Inspired by the biopsychosocial model introduced by Engel in 1977, which tried to overcome the drawbacks and limitations of the dualistic biomedical model, Loeser ${ }^{4}$ and Waddell ${ }^{5}$ both established modified versions of the biopsychosocial model adapted to the context of pain. Pain becomes a multidimensional concept (nociception, pain, suffering, and pain behavior) arising from multiple etiological factors and complex interactions. ${ }^{6}$

The prevalence of chronic pain is highly variable and ranges from $10 \%$ to $64 \%$, depending on the studied population, the study design, and the clinical criteria used. ${ }^{7,8}$ Chronic pain imposes a tremendous burden, both in terms of costs and quality of life, affecting and deeply involving not only the patient but also their family, caregivers, and peers in general. Living with chronic pain implies facing and coping with serious challenges, as it seriously interferes with normal physical, social, and physiological functions and with daily work activities. ${ }^{9}$

Various psychosocial variables have been shown to be associated with chronic pain experience and perception: female sex, ${ }^{10}$ low socioeconomic status, low educational level, lack of personal and social support, family history of chronic pain, cultural and ethnic identity, ${ }^{11}$ and immigrant status. $^{10,12,13}$

Bates et al ${ }^{14,15}$ have particularly explored the topic of ethnic identity in pain perception and experience, using both quantitative and qualitative approaches, and have identified that subjects from New England and Puerto Rico experienced chronic pain differently.

The extant literature shows that the prevalence of chronic pain is higher among immigrants than among the native population of a country, even though not all authors agree with this view, claiming that the so-called Mediterranean syndrome is a myth lacking solid evidence. ${ }^{16}$ However, a recently published Danish investigation, the Danish Health Study, found that individuals with a non-western background had a significantly higher risk of reporting chronic pain than those with a Danish background. ${ }^{17}$ The Zurich Study, an investigation carried out in Switzerland, found high levels of pain intensity, depression, anxiety, and catastrophizing at baseline in a group with an immigration background compared with a group without this background. ${ }^{18}$ A study carried out in the USA showed a link between acculturation and chronic pain among Latino Americans. ${ }^{19}$ In a study carried out in Sweden, immigrant status was statistically associated with chronic pain behavior, with an odds ratio of $1.83 .{ }^{12}$

Therefore, immigrant women represent a particularly vulnerable group. ${ }^{9}$ In such groups, coping strategies play a major role in reducing the burden of anxiety and depression related to the immigration background and new context, as shown in some studies. Cultural self-efficacy was shown to act as a mediator in mitigating altered mental health in a sample of Chinese immigrants, ${ }^{20}$ as well as social support. ${ }^{21}$ Social capital (in Chinese guanxi) is particularly valuable in Chinese communities. ${ }^{22}$

In this study, we chose to focus on Chinese women in Italy, as they represent a particular group of immigrants who, differently from others, do not use the local health care systems, but prefer to use traditional Chinese medical systems. ${ }^{23}$ The Chinese population in Italy numbers about 270,000 (5.3\% of the entire population of immigrants) and $49.0 \%$ of them are women. ${ }^{24}$ It is a population that is not adequately covered by the extant literature. For example, in various surveys performed by Naga, an important Italian voluntary association that offers free medical care to immigrants, Chinese communities are underrepresented, as such research tends to include subjects who trust Western medicine. ${ }^{25,26}$ Very little is known about how Chinese women in Italy live with and face chronic pain. The present pilot research was aimed at starting to fill this gap of knowledge.

In particular, our study aimed to examine the burden of chronic pain in Chinese immigrants in Italy in terms of their acculturation processes, perceived control over disease (locus of control, self-efficacy), social networks, and coping strategies. The following research questions were investigated:

1. How do acculturation strategies affect the orientation and adoption of healthy behaviors and lifestyles? How does the acculturation level influence the choice of managing the disease ${ }^{27}$ and, in particular, treating the chronic pain?

2. How does perception of control over disease (internal versus external locus of control, self-efficacy) influence the adoption of healthy behaviors and the use of traditional versus conventional medicine in migrant communities ${ }^{28}$

3. Does the level of contact that the subject has with the country of origin and their interactions with the host community influence the adoption of new beliefs and new behaviors in terms of health and disease? Or does it 
allow the maintenance of originally learned and acquired concepts and health strategies?

4. Are coping strategies for chronic pain in a migrant community culturally influenced ${ }^{29}$

\section{Methods}

We performed a thematic field analysis, one of the most common approaches used in the field of qualitative research together with discourse/conversation analysis, grounded analysis, and interpretative phenomenological/hermeneutic analysis, among others. ${ }^{30,31}$ This type of approach leads to the emergence of meaningful patterns (known as themes), which can be both implicit or explicit ideas. These are identified by developing proper codes, which are later reviewed to ensure that no other themes are missing and that the whole text has been covered and adequately mapped. This implies a complex, iterative process of extensively reading and rereading texts to become familiar with them and then coding, examining, recording, reviewing, and analyzing the themes, as well as thematic variations, associated with our research questions.

A sample of Chinese women from six Italian cities (Turin, Florence, Prato, Bologna, Genoa, and Milan) was recruited via snowballing from various Chinese migrant communities, associations, and hospitals. The inclusion criteria were 1) age $\geq 18$ years; 2 ) having chronic pain; and 3 ) being able to speak Italian. In total, 82 Chinese women volunteered to take part in this study (Figure 1), and they were interviewed by means of a semi-structured questionnaire during the period of May 2014-March 2015. The questionnaire investigated biographical data and information about migration history; acculturation and acculturation processes in terms of nearness and distance between the migrant and the belonging culture and the host culture in daily life (food, language, relationship with China) and in chronic pain experience (use of traditional Chinese medicine [TCM]) versus conventional modern Western medicine) $;{ }^{32-34}$ perception and feelings of the migrant about pain and, in particular, chronic pain; coping strategies adopted in the chronic pain situation; and, finally, the presence of social support in the chronic pain situation. For further details, see Figure 2.

In-depth interviews (average 1 hour) were performed and then transcribed by one of the authors (TSR) and processed using ATLAS.ti qualitative analysis software (version 7.0; ATLAS.ti GmbH, Berlin, Germany). Frequency and cooccurrence analyses were carried out. To ensure that no meaning was lost during the process, every transcribed quotation was analyzed and discussed by four authors (TSR, NLB, $\mathrm{SF}$, and $\mathrm{CCP}$ ) who were content and methodology experts.

This study was approved by the Human Subjects Review Committee of the University of Genoa and by the UNESCO Chair "Health Anthropology, Biosphere and Healing Systems". Written informed consent was obtained from all participants in the study. The study was conducted in accordance

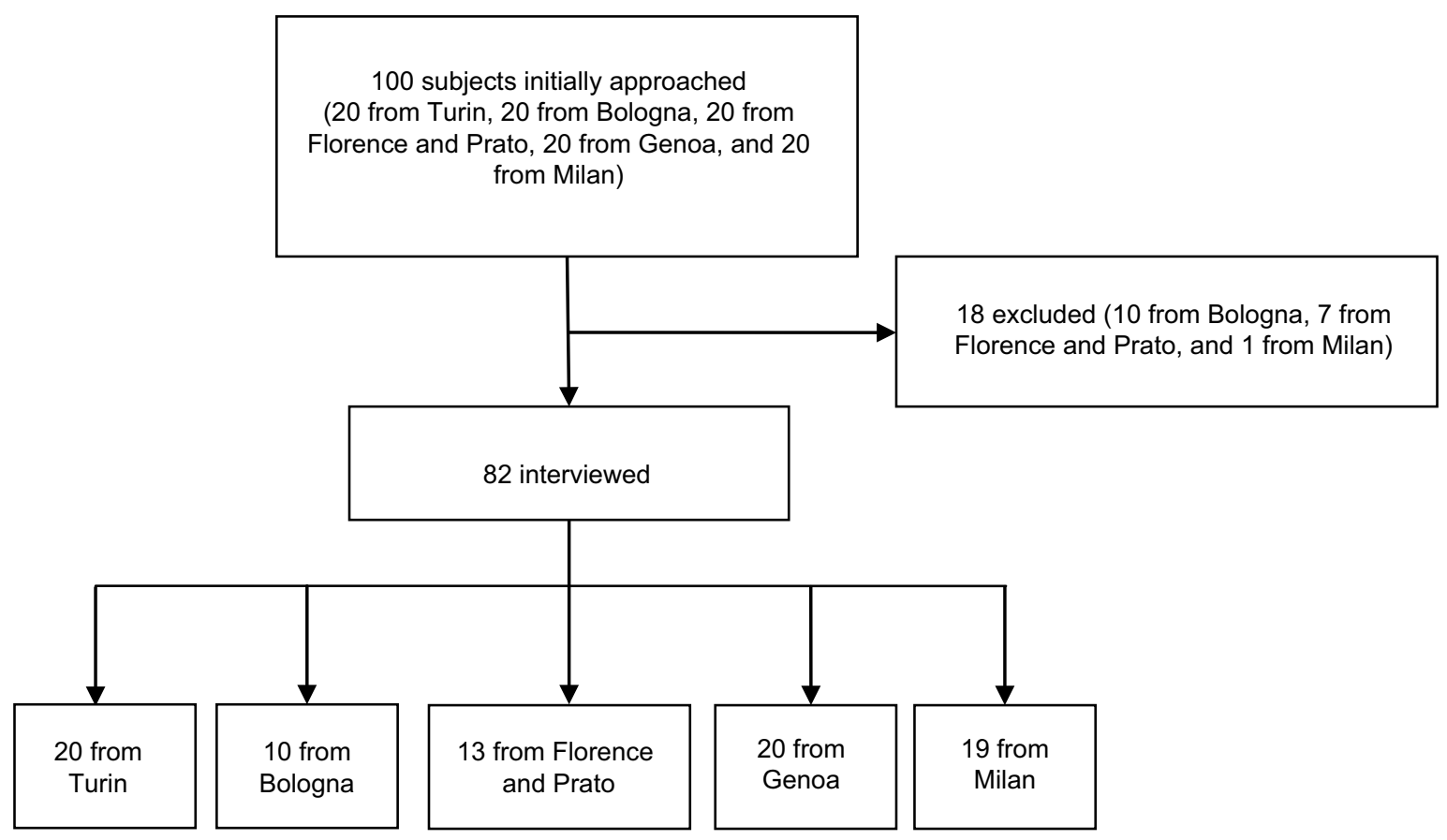

Figure I Flowchart of subjects who met inclusion/exclusion criteria for the study population. 


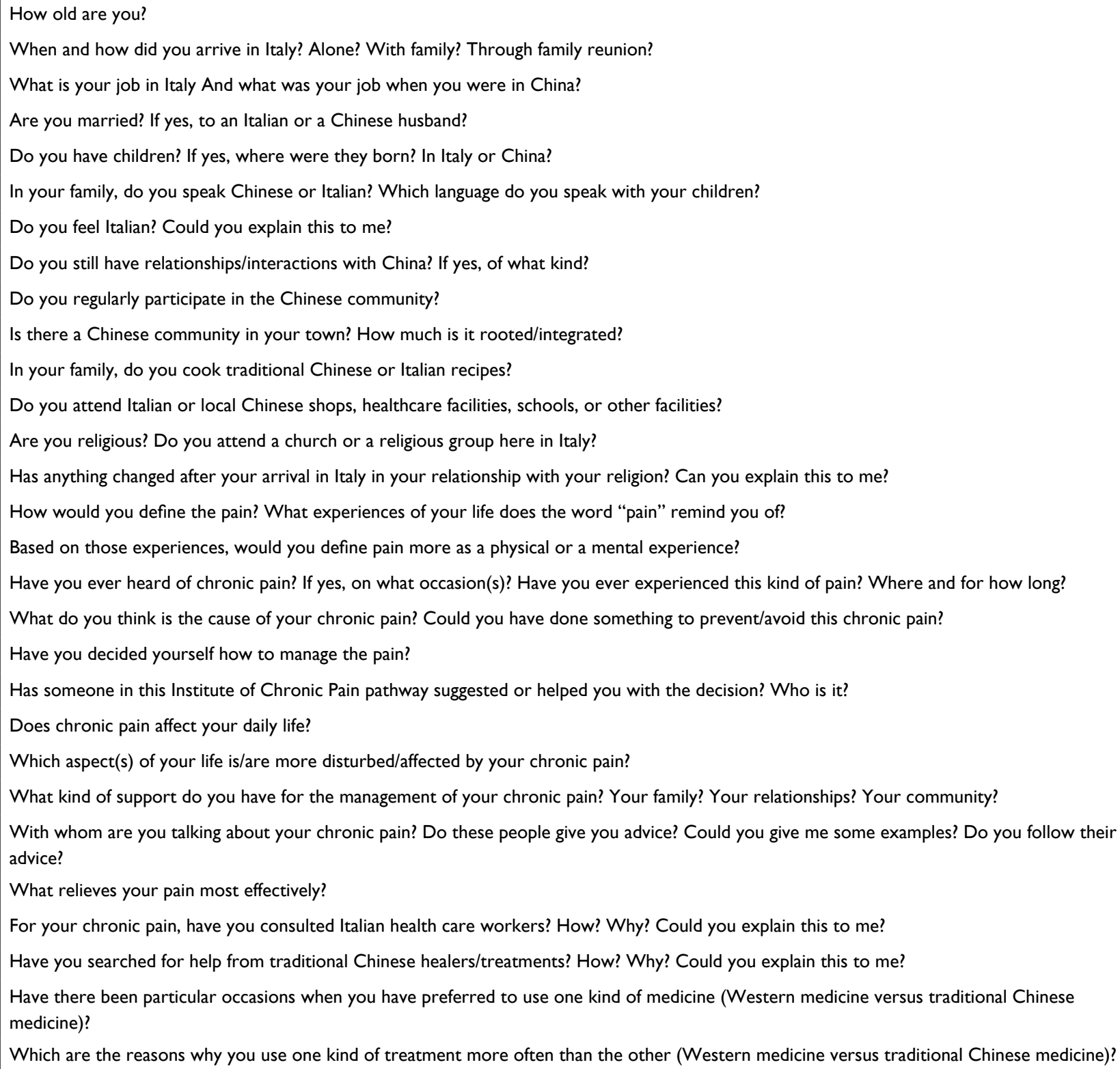

Figure 2 List of the questions contained in the semi-structured questionnaire.

with the ethical principles for medical research proclaimed by the Declaration of Helsinki and its amendments.

\section{Results}

Our findings underline the tremendous burden of chronic pain, which affected all aspects of the women's lives. The sociodemographic characteristics of our sample are reported in Table 1. Most of them had chronic musculoskeletal pain.

\section{Acculturation processes}

Overall, the sense of belonging to the host culture was strong:
I love this country, I love its people, and cuisine, above all. I regularly attend the Chinese community in Milan, the historic association in Via Paolo Sarpi, but, together with my husband, I also have Italian friends that we have known here, in Italy. Sometimes, they visit us in our house, sometimes we go out with them. Sara cooks very well and tries to teach me some Italian recipes. I do the same with her with some Chinese recipes. [Milan; subject ID 4]

However, this strong sense of belonging did not simply reflect or translate into a linear engagement with the medical system, as the health care pathways were more complex and dual, as also reported by Green et al. ${ }^{34}$ For Chinese women 
Table I Sociodemographic variables of the recruited sample

\begin{tabular}{ll}
\hline Sociodemographic variables & Results (\%) \\
\hline Arrival in Italy & \\
With family & 25.60 \\
Alone & 24.40 \\
Through family reunion & 50.00 \\
Time of arrival in Italy & \\
I990 or earlier & 13.40 \\
I99I-2000 & 25.60 \\
200I-20I5 & 48.80 \\
Born in Italy & 12.20 \\
Age range, years & \\
I8-45 & 67.00 \\
46-65 & 33.00 \\
Marital status & \\
Married to a Chinese & 56.30 \\
Married to an Italian & 7.30 \\
Not married & 15.80 \\
Engaged to a Chinese & 9.70 \\
Engaged to an Italian & 10.90 \\
With children & 63.50 \\
Occupation & \\
Student & 23.10 \\
Unemployed & 4.80 \\
Employed & 46.50 \\
Self-employed & 25.60 \\
\hline
\end{tabular}

who were not fully integrated into the Italian culture and who spoke Italian less fluently, access to the Italian health care system was characterized by perceived barriers, especially in establishing an effective therapeutic alliance. In addition, even Chinese women who reported that they feel strongly and deeply rooted in the Italian environment did not discontinue the use of TCM and continued to engage with Chinese remedies. This was particularly true for women who reported that conventional Western medicine was ineffective and/or caused adverse effects. Generally speaking, Chinese women preferred to use traditional Chinese remedies (such as acupuncture or moxibustion) rather than conventional medicine. However, the Italian health care system was preferred during pregnancy.

I have just had my acupuncture, because I have a terrible backache. [Turin, subject ID 8]

Furthermore, some Chinese women articulated their difficulties in accessing the Italian medical system in that their health needs were not properly recognized and addressed. The use of TCM was also suggested and encouraged by social networks.

My husband advises me not to go the Italian doctors. [FI 10] My husband suggested I should go to a Chinese doctor and I did. [Genoa, subject ID 2]

\section{Living with chronic pain}

For immigrants with chronic pain, the pain became the main focus of their life and extensively and adversely affected their daily life, interfering particularly with work.

It seems everlasting. I feel myself consumed by the pain.

[Turin, subject ID 5]

Chronic pain alters mental health and causes sleepiness and anxiety.

After the death of my husband, my pain has worsened, I do not manage to sleep anymore. [Turin, subject ID 11]

\section{Coping strategies}

The interviewed Chinese women used a variety of coping strategies to manage the pain, as well as its consequences and impact on daily life and work. Younger women were more likely than older women to use proactive coping. Overall, the coping strategies used were mainly adaptive behaviors, being problem focused or maladaptive, relying upon "cope and avoid" mechanisms.

Reducing daily activities and especially working less were not perceived as possible solutions, as Chinese women reported that work is extremely important in the Chinese culture.

I could try to reduce my working hours, but work is so important for us. In China, someone who does not work is not well thought of and this for us is terrible. [Bologna, subject ID 2]

Some women even introduced the theme of sociocultural stigma, ie, the fear of being discriminated against by the other members of the community if they reduced their work activities in order to mitigate the burden of chronic pain. This emphasizes the relevance of work to the Chinese culture.

\section{Self-efficacy}

Self-efficacy, defined as the extent or strength of the belief of these Chinese women to be able to cope with their chronic pain, was particularly evident when the Western medicine was ineffective and they proactively searched a remedy, looking at TCM as an alternative. However, self-efficacy did not result in higher compliance with or adherence to TCM or in changes in lifestyle behaviors. This was perceived and interpreted as an autonomous choice and decision and as a way for the patients to assert agency.

I could not bear standing and working so many hours. I decided to go to the doctor. I don't know why I suffer from this terrible pain. My mother does not, and also my father 
has never reported such pain, as well as none of my family. I have a terrible pain here [...]. [Turin, subject ID 11]

\section{Locus of control}

Perceived control over chronic pain was usually external. Chinese women perceived pain as physical rather than psychological and attributed its causes to external factors rather than to their lifestyles or their migration history and background.

It is due to too much work. And it worsened after the death of my husband. [Genoa, subject ID 2]

\section{Social support}

Younger women engaged with formal social networks, while older women preferred informal support. Overall, Chinese women experiencing chronic pain benefited from social networks and support, which were mainly informal and based on Chinese peers: usually parents, friends, or people from Chinese communities and associations.

My family helps me a lot when the pain interferes with my daily and work activities. In particular, I receive a great amount of support from my children, which helps me in almost everything. [Florence, subject ID 5]

This bond was perceived as particularly effective in mitigating the burden related to chronic pain. Thus, the stock of social network became resource rich and goal oriented and, as such, was perceived as a social bonding capital.

\section{Discussion}

The present study investigated the impact of acculturation, locus of control, coping strategies, and self-efficacy on chronic pain in Chinese women who were immigrated to Italy.

We found a high level of acculturation in our sample. This finding contrasts with some cross-cultural studies that show that Chinese immigrants are more likely to encounter acculturative stress than other immigrants. ${ }^{35}$ Our finding could be explained by the fact that our sample was mostly made up of young women who tend to exhibit more "resilient adaptability" than young men and older women. ${ }^{36}$

Our sample perceived chronic pain as being physical rather than mental or psychophysical. This is in agreement with the observation that immigrants tend to report somatic symptoms - the so-called somatization hypothesis. ${ }^{37}$

We found that active coping was not particularly exploited by Chinese women with chronic pain. This is confirmed by the extant literature: a number of studies have shown that immigrants tend to report low scores for active coping strategies. ${ }^{9,38}$

Our findings concerning self-efficacy also confirm those found by Michaëlis et al. ${ }^{9}$ Non-Western immigrants in Denmark perceived and felt that actively seeking health care was an effective means to assert one's own identity. Wu and Zanin $^{23}$ emphasized that many Chinese immigrants found their health needs unmet and that this led to the establishment of various unofficial Chinese clinics.

The locus of control was found to be external in the current study. This is in agreement with findings from the literature, which show that immigrants tend to have more external perceived control than nonimmigrants. ${ }^{39}$ Some studies have further linked external locus with depressive symptoms. ${ }^{40}$

Social networks, conceptualized as social capital and categorized as social bonding capital, played an important role in mitigating the burden of chronic pain and even in suggesting new medical pathways that are alternatives to conventional Western health care pathways. This is in line with what was found by Devillanova ${ }^{26}$ in undocumented immigrants in Milan, who were referred to a certain medical pathway or to another health care opportunity by a strong social tie (parents, relatives, or friends).

The finding of underuse of the health service by the Chinese migrant community is in line with the extant literature. ${ }^{25,26,41-45}$ Further, immigrants tend to use emergency services rather than attending specialists or using preventive services. ${ }^{43}$

Our study has a particular strength in that it focuses on a migrant community usually underrepresented in literature surveys and research; however, it has a number of limitations that need to be acknowledged. These are mainly due to the sampling strategy used and the language difference. Although conducting interviews with interpreters was possible, we preferred to include only Chinese women able to speak Italian because we felt that otherwise the presence of the interpreter might have affected the responses. However, this could represent a further selection bias of the sample.

As far as the sampling strategy is concerned, undoubtedly the presence of Chinese immigrants in Italy is more complex and heterogeneous in terms of health needs and use of medical systems, as well as in terms of language skills, working patterns, job mobility, and attitude, among others. ${ }^{23}$ Some Chinese immigrants are indeed integrated into the Italian society, whereas others live in underground realities and contexts and are particularly difficult to reach, having irregular and complex interactions with the Italian system. ${ }^{42,43}$ 


\section{Conclusion}

The extant literature on immigrants with chronic pain is mostly quantitative. Our findings underline the importance of carrying out qualitative research, from which the tremendous burden of chronic pain emerges and is seen to affect all aspects of the women's lives. Health care workers and providers should be aware of the complexity of chronic pain, resulting from an array of different variables. Therefore, as advocated by Michaëlis et al, ${ }^{9}$ a culturally sensitive approach should be adopted when managing chronic pain and approaching immigrant patients, taking into particular account their ethnic diversity ${ }^{33}$ and therefore adopting a cultural lens and an emic perspective ${ }^{14}$ within a holistic framework. This should consider the involvement of the different stakeholders, promotion of multidimensional interventions, facilitation of health care access, removal of bureaucratic/administrative obstacles, and favor of socially inclusive policies.

However, owing to the aforementioned limitations of the current study, further research in this field is urgently needed.

\section{Disclosure}

The authors report no conflicts of interest in this work.

\section{References}

1. Loeser JD, Treede RD. The Kyoto protocol of IASP Basic Pain Terminology. Pain. 2008;137(3):473-477.

2. Turk DC, Okifuji A. Pain terms and taxonomies. In: Loeser D, Butler SH, Chapman JJ, Turk DC, editors. Bonica's Management of Pain. 3rd ed. Philadelphia, PA: Lippincott Williams \& Wilkins; 2001:18-25.

3. Chrubasik S, Junck H, Zappe HA, Stutzke O. A survey on pain complaints and health care utilization in a German population sample. Eur J Anaesthesiol. 1998;15:397-408.

4. Loeser JD. Concepts of pain. In: Stanton-Hicks J, Boaz R, editors. Chronic Low Back Pain. New York: Raven Press; 1982:109-142.

5. Waddell G. A new clinical method for the treatment of low back pain. Spine. 1987;12:632-644.

6. Gatchel RJ, Peng YB, Peters ML, Fuchs PN, Turk DC. The biopsychosocial approach to chronic pain: scientific advances and future directions. Psychol Bull. 2007;133:581-624.

7. Häuser W, Wolfe F, Henningsen P, Schmutzer G, Brähler E, Hinz A. Untying chronic pain: prevalence and societal burden of chronic pain stages in the general population - a cross-sectional survey. BMC Public Health. 2014;14:352.

8. Harstall C, Ospina M. How prevalent is chronic pain? Pain Clin Updates. 2003;11(2):1-4.

9. Michaëlis C, Kristiansen M, Norredam M. Quality of life and coping strategies among immigrant women living with pain in Denmark: a qualitative study. BMJ Open. 2015;5:e008075.

10. Bergman S. Psychosocial aspects of chronic widespread pain and fibromyalgia. Disabil Rehabil. 2005;27:675-683.

11. Bates MS, Edwards WT. Ethnic variations in the chronic pain experience. Ethn Dis. 1992;2:63-83.

12. Bergman S, Herrström P, Högström K, Petersson IF, Svensson B, Jacobsson LT. Chronic musculoskeletal pain, prevalence rates, and sociodemographic associations in a Swedish population study. J Rheumatol. 2001;28(6):1369-1377.
13. Löfvander MB, Furhoff AK. Pain behaviour in young immigrants having chronic pain: an exploratory study in primary care. Eur J Pain. 2002;6(2):123-132.

14. Bates MS, Rankin-Hill L, Sanchez-Ayendez M, Mendez-Bryan R. A cross-cultural comparison of adaptation to chronic pain among Anglo-Americans and native Puerto Ricans. Med Anthropol. 1995;16(2):141-173.

15. Bates MS, Rankin-Hill L, Sanchez-Ayendez M. The effects of the cultural context of health care on treatment of and response to chronic pain and illness. Soc Sci Med. 1997;45(9):1433-1447.

16. Ernst G. The myth of the 'Mediterranean syndrome': do immigrants feel different pain? Ethn Health. 2000;5(2):121-126.

17. Kurita GP, Sjøgren P, Juel K, Højsted J, Ekholm O. The burden of chronic pain: a cross-sectional survey focussing on diseases, immigration, and opioid use. Pain. 2012;153(12):2332-2338.

18. Kellner U, Halder C, Litschi M, Sprott H. Pain and psychological health status in chronic pain patients with migration background--the Zurich study. Clin Rheumatol. 2013;32(2):189-197.

19. Bui Q, Doescher M, Takeuchi D, Taylor V. Immigration, acculturation and chronic back and neck problems among Latino-Americans. J Immigr Minor Health. 2011;13(2):194-201.

20. Du H, Li X, Lin D, Tam CC. Collectivistic orientation, acculturative stress, cultural self-efficacy, and depression: a longitudinal study among Chinese internal migrants. Community Ment Health J. 2015;51(2):239-248.

21. Lee EH, Zhou Q, Ly J, Main A, Tao A, Chen SH. Neighborhood characteristics, parenting styles, and children's behavioral problems in Chinese American immigrant families. Cultur Divers Ethnic Minor Psychol. 2014;20(2):202-212.

22. Huang J, Aaltio I. Guanxi and social capital: networking among women managers in China and Finland. Int J Intercult Relat. 2014;39:22-39.

23. Wu B, Zanin V. Discussion Paper 48. Healthcare Needs of Chinese Migrant Workers in Italy: A Survey Report on Chinese-Owned Workshops in Veneto. The University of Birmingham, China Policy Institute; 2009. Available from: https://www.nottingham.ac.uk/cpi/documents/ discussion-papers/discussion-paper-48-healthcare-chinese-migrantsitaly.pdf. Accessed January 6, 2016.

24. ISTAT. Immigrants in Italy. 2015. Available from: http://www.istat.it/ it/archivio/immigrati. Accessed January 6, 2016.

25. Devillanova C. Immigrants' Access to Health Care Services in Italy: New Evidence from Survey Data. 2016. Available from: http://www.siecon. org/online/wp-content/uploads/2012/08/Devillanova.pdf. Accessed July $13,2016$.

26. Devillanova C. Social networks, information and health care utilization: evidence from undocumented immigrants in Milan. $J$ Health Econ. 2008;27:265-286.

27. Lasch KE. Culture, pain, and culturally sensitive pain care. Pain Manag Nurs. 2000;1(3 suppl 1):16-22.

28. Chan MY, Hamamura T, Janschewitz K. Ethnic differences in physical pain sensitivity: role of acculturation. Pain. 2013;154(1):119-123.

29. Ferreira-Valente MA, Pais-Ribeiro JL, Jensen MP. Associations between psychosocial factors and pain intensity, physical functioning, and psychological functioning in patients with chronic pain: a cross-cultural comparison. Clin J Pain. 2014;30(8):713-723.

30. Clarke V, Braun V. Teaching thematic analysis: overcoming challenges and developing strategies for effective learning. Psychologist. 2013;26(2):120-123.

31. Friese S. Qualitative Data Analysis with ATLAS.ti. Thousand Oaks, CA: SAGE Publications; 2014

32. Berry JW. Acculturation: living successfully in two cultures. Int J Intercult Relat. 2003;29(2005):697-712.

33. Edrington J, Sun A, Wong C, et al. A pilot study of relationships among pain characteristics, mood disturbances, and acculturation in a community sample of Chinese American patients with cancer. Oncol Nurs Forum. 2010;37(2):172-181.

34. Green G, Bradby H, Chan A, Lee M. "We are not completely Westernised": dual medical systems and pathways to health care among Chinese migrant women in England. Soc Sci Med. 2006;62(6):1498-1509. 
35. Pan JY, Wong DF. Acculturative stressors and acculturative strategies as predictors of negative affect among Chinese international students in Australia and Hong Kong: a cross-cultural comparative study. Acad Psychiatry. 2011;35(6):376-381.

36. Kwak K, Rudmin F. Adolescent health and adaptation in Canada: examination of gender and age aspects of the healthy immigrant effect. Int J Equity Health. 2014;13:103.

37. Bragazzi NL, Del Puente G, Natta WM. Somatic perception, cultural differences and immigration: results from administration of the Modified Somatic Perception Questionnaire (MSPQ) to a sample of immigrants. Psychol Res Behav Manag. 2014;7:161-166.

38. Hjellset VT, Ihlebæk CM, Bjørge B, Eriksen HR, Høstmark AT. Healthrelated quality of life, subjective health complaints, psychological distress and coping in Pakistani immigrant women with and without the metabolic syndrome: the InnvaDiab-DEPLAN Study on Pakistani immigrant women living in Oslo, Norway. J Immigr Minor Health. 2011;13(4):732-741.

39. Magwaza AS, Bhana K. Stress, locus of control, and psychological status in black South African migrants. J Soc Psychol. 1991;131(2): 157-164.
40. van Dijk TK, Dijkshoorn H, van Dijk A, Cremer S, Agyemang C. Multidimensional health locus of control and depressive symptoms in the multi-ethnic population of the Netherlands. Soc Psychiatry Psychiatr Epidemiol. 2013;48(12):1931-1939.

41. Aroian KJ, Wu B, Tran TV. Health care and social service use among Chinese immigrant elders. Res Nurs Health. 2005;28(2):95-105.

42. Ma GX. Between two worlds: the use of traditional and Western health services by Chinese immigrants. J Community Health. 1999;24(6):421-437.

43. Lai DW, Chau SB. Effects of service barriers on health status of older Chinese immigrants in Canada. Soc Work. 2007;52(3):261-269.

44. Lai DW, Chau SB. Predictors of health service barriers for older Chinese immigrants in Canada. Health Soc Work. 2007;32(1):57-65.

45. Giannoni M, Ismail Z. Rassegna di letteratura sulle disuguaglianze di salute e accesso ai servizi sanitari relative alla popolazione immigrata in Italia. [A literature review of inequities in healthcare services among immigrants in Italy] In: Giannoni M, editor. Equità Nell'accesso Ai Servizi Sanitari, Disuguaglianze Di Salute E Immigrazione. La Performance Dei Servizi Sanitari [Equity in Access to Healthcare Services, Inequities in Health and Immigration. Performances of Health Systems]. Milan, Italy: Franco Angeli; 2010.
Journal of Pain Research

\section{Publish your work in this journal}

The Journal of Pain Research is an international, peer reviewed, open access, online journal that welcomes laboratory and clinical findings in the fields of pain research and the prevention and management of pain. Original research, reviews, symposium reports, hypothesis formation and commentaries are all considered for publication.

\section{Dovepress}

The manuscript management system is completely online and includes a very quick and fair peer-review system, which is all easy to use. Visit http://www.dovepress.com/testimonials.php to read real quotes from published authors. 